\title{
The role of T-lymphocyte subsets and interleukin-5 blood levels among Indian subjects with autoimmune thyroid disease
}

\author{
Sripathy Gopalakrishnan ${ }^{1}$, Sourav Sen ${ }^{2}$, Jawahar Singh Adhikari ${ }^{3}$, \\ Pradeep Kumar Chugh ${ }^{3}$, Tarun Sekhri ${ }^{3}$, Sankar Rajan ${ }^{4}$ \\ ${ }^{1}$ Defence Bio-engineering and Electromedical Laboratory, Bangalore, ${ }^{2}$ Armed Forces Medical College, Pune, ${ }^{3}$ Institute of \\ Nuclear Medicine and Allied Sciences, Timarpur, Delhi, ${ }^{4}$ Global Alliance for Improved Nutrition, New Delhi, India
}

\begin{abstract}
OBJECTIVE: Autoimmune Thyroid Disease (AITD) results from an interaction of exogenous and endogenous factors in a genetically predisposed individual. AITD is being increasingly reported among the Indian population. Lymphocyte subsets and levels of interleukin-5 (IL5) were studied in the peripheral blood of patients with AITD. DESIGN: Subjects diagnosed with either hyperthyroidism due to Graves' Disease (GD) or with primary hypothyroidism due to Hashimoto's Thyroiditis (HT) were consecutively recruited. Euthyroid controls were also recruited for comparison. Lymphocyte subsets (CD4 and CD8 counts, CD4/CD8 ratio) were evaluated by flow cytometry and IL-5 levels were determined by the sandwich ELISA method. RESULTS: Nineteen subjects with GD, 16 subjects with HT and 10 controls were studied. CD4/CD8 ratios were found to be significantly lower only in subjects with HT compared to controls. Serum IL-5 values were significantly higher in both GD and HT in comparison to controls. CONCLUSIONS: The study found increased levels of IL-5 and reduction in ratios of CD4/CD8 lymphocytes in the peripheral blood of patients with HT, but only IL-5 was increased in GD. High levels of IL-5 could have resulted in the high titres of antithyroid antibodies and may therefore be considered to play a more significant role than peripheral lymphocytes in the pathogenesis of AITD in the Indian population.
\end{abstract}

Key words: Graves' hyperthyroidism, Hashimoto's thyroiditis, Hypothyroidism, Interleukin-5, CD4/CD8 ratio, Anti-thyroid antibodies

Address for correspondence:

Dr. Sripathy G, Defence Bio-engineering and Electromedical Laboratory, Post Bag No: 9326, CV Raman Nagar, Bangalore560096, e-mail: gkaustubh@gmail.com

Received 05-08-09, Revised 10-10-09, Accepted 20-11-09

\section{INTRODUCTION}

Autoimmune Thyroid Disease (AITD) constitutes a spectrum of thyroid disorders, the two extremes being Graves' Disease (GD), characterized by 
hyperthyroidism, and Hashimoto's Thyroiditis (HT), characterized by hypothyroidism. ${ }^{1,2}$ The pathogenesis of thyroid autoimmunity is thought to be multifactorial, with contributions from both humoral as well as cell-mediated immune systems. ${ }^{3}$ This generalized view has been further refined by recent advances in immunology which show that autoimmune response in AITD is more focused, involving a restricted number of B-cells and T-cells, while cytokines released in the process are also involved in the pathogenesis. ${ }^{4,5}$ The cell-mediated immune process is now known to be mediated by CD4+ and CD8+ lymphocyte subsets of T-cells which have a major role in AITD. ${ }^{6}$ Among the differentiated CD4+ T-cells, two functionally distinct subsets have been identified based on their cytokine secretion profiles, namely Type 1 and Type 2 (Th1 \& Th2). ${ }^{7}$ Th2 cells secrete the interleukins IL-4, IL-5, IL-6, among others, and promote production of antibodies. Antithyroid antibodies, namely Antimicrosomal (AMc), anti-thyroglobulin (ATg) and anti-TSH receptor (TRAb) antibodies, are associated with HT and GD. ${ }^{3,8}$ Consequently, differences in the pattern of cytokine secretion, especially that of IL-5 and IL-12, were further studied to identify a possible role of these cytokines in the pathogenesis of AITD. ${ }^{9-11}$

Thyroid autoimmunity is being increasingly identified as the underlying cause of goitre in India subsequent to the implementation of the salt iodization program in the country. ${ }^{12}$ Meanwhile, there is a paucity of literature on the immunopathogenesis of AITD among the Indian population. A study was carried out at the thyroid clinic of the Institute of Nuclear Medicine and Allied Sciences (INMAS), Delhi, to evaluate the relationship between AITD with lymphocyte subsets (CD4/CD8 ratio) and cytokine interleukin-5 (IL-5).

\section{MATERIALS AND METHODS}

\section{Subjects and study protocol}

The thyroid clinic of the INMAS is a tertiary level referral centre with patients referred from all over north India. Adult subjects in the age range of 20-60 years who were diagnosed for the first time with either hyperthyroidism from Graves' disease or hypothyroidism from Hashimoto's thyroiditis at the
INMAS were recruited consecutively into the study. Informed consent was obtained from all. Control subjects were recruited after taking a history to rule out thyroid illness in the past, clinical examination and the recording of biochemical parameters (thyroid hormones and thyrotropin). The controls were clinically and biochemically euthyroid and were negative for antithyroid antibodies. The study was approved by the research advisory panel of the Defence Research and Development Organisation under the Ministry of Defence, Government of India.

Diagnosis of AITD was made after clinical examination and pertinent investigations [serum thyroxine (T4), serum thyrotropin (TSH), AMc, radioactive iodine uptake (RAIU)] based on a protocol. Diagnostic criteria for Graves' disease were clinical features of thyrotoxicosis, elevated thyroid hormones in blood with suppressed TSH and elevated RAIU. Hypothyroidism from HT was diagnosed in subjects with diffuse goitre who were found to have elevated titres of antimicrosomal antibodies in serum, serum T4 lower than normal range and elevated TSH.

Whole blood samples were drawn after overnight fasting from all the recruited patients and controls. These were analyzed for lymphocyte counts, CD4/CD8 ratios and cytokine interleukin-5 (IL-5). Results were collected and data was evaluated using the statistical software SPSS version 15.0 (SPSS Inc., Chicago, USA). Numerical data are expressed as mean \pm standard deviation (SD). Differences in IL-5 and subset ratios between the groups were analyzed using the KruskalWallis and two-sample Kolmogorov-Smirnov test, while correlation of these parameters with other parameters within each group was carried out using Spearman's Rho test. Differences were considered to be statistically significant if $\mathrm{p}$ values were $<0.05$.

\section{Laboratory methods}

\section{Method for CD4 / CD8 counts using flow cytometry}

Whole blood samples of all the subjects were first stained with Reagents A through F. Diluted Reagent $\mathrm{G}$ is used to lyse erythrocytes following staining. The procedure was performed at room temperature $\left(20^{\circ}\right.$ to $25^{\circ} \mathrm{C}$ ) using reagents, under a predetermined protocol. The samples were incubated, and centrifuged 
and supernatant was aspirated. The remaining cell pellet was resuspended and $0.1 \%$ sodium azide was added to each tube. After further centrifugation, the samples were prepared for flow cytometry. A flow cytometer was first prepared for sample analysis using CaliBRITE beads and AutoCOMP software. The stained samples were then run on the flow cytometer and analyzed with Simultest IMK-Lymphocyte software. ${ }^{13}$ Patient data were stored in list mode to allow subsequent analysis of data files. The software automatically collects a sufficient number of events to obtain a minimum of 2,000 lymphocytes within the lymphocyte gate.

\section{Cytokine assay}

Serum levels of interleukin-5 were measured using a sandwich enzyme-linked immunosorbent assay similar to the method already described (Wako Pure Chemical Industries, Osaka, Japan). ${ }^{10}$ All assays were done in duplicate. The detection limit of the assay was $4 \mathrm{pg} / \mathrm{ml}$ and the intra-assay and inter-assay coefficients of variation were $7 \%$ and $12 \%$, respectively.

\section{Thyroid function and antibody tests}

Serum thyroxine (T4) and thyrotropin (TSH) were estimated by radioimmunoassay (RIA) and immunoradiometric assay (IRMA), respectively. The kits were obtained from the Bhabha Atomic Research Centre, Mumbai, India. The reference range of normal values for T4 is 4.5-12.5 $\mu \mathrm{g} / \mathrm{dl}^{*}$ and for TSH is $0.25-5.0$ $\mu \mathrm{IU} / \mathrm{ml}$. Antithyroid antibodies (AMc) and ATg were determined by commercially available semi-quantitative hemagglutination (Serodia) kits obtained from Fujerobio Inc, Tokyo, Japan. Tests were considered positive only if titres were higher than 1:400.

\section{RESULTS}

In the final analysis there were three groups of subjects. The first group, namely "GD", consisted of 19 patients with Graves' hyperthyroidism with a mean age of 37.1 years $(\mathrm{SD}=10.5$ years), the second group named "HT" was composed of 16 hypothyroid patients with Hashimoto's thyroiditis whose mean age was 34.9 years $(\mathrm{SD}=11.2$ years) and the third group consisted of 10 euthyroid controls with a mean age of 32.6 years ( $\mathrm{SD}=10.2$ years). The characteristics of the patient and euthyroid control population are given in Table 1.

\section{CD4/CD8 ratio}

The ratio was $1.50 \pm 0.55$ (mean $\pm \mathrm{SD}$, range: 0.58 2.4 ) in patients with GD, $1.10 \pm 0.32$ (mean \pm SD, range: $0.54-1.67$ ) in patients with $\mathrm{HT}$ and $2.12 \pm 0.76$ (mean \pm SD, range: $0.74-3.43$ ) in euthyroid subjects. When the Kolmogorov-Smirnov test (KS test) was applied to the ratios, results showed that the values for HT were significantly lower compared to those of the control group $(\mathrm{p}<0.01)$. When the values of GD were compared with the HT and control groups using the KS test, the difference was not found to be statistically significant ( $p>0.01)$.

Table 1. Characteristics of the study population

\begin{tabular}{|c|c|c|c|c|c|}
\hline S. No & Characteristics & Euthyroid controls & HT with hypothyroidism & Graves' hype & thyroidism \\
\hline 1. & Number (n) & 10 & 16 & 19 & \\
\hline \multirow[t]{2}{*}{2.} & Age (years) & & & & \\
\hline & Mean (SD) & $32.6(10.2)$ & $34.9 \quad(11.2)$ & 37.1 & $(10.5)$ \\
\hline 3. & Males/females & $2 / 8$ & $3 / 13$ & $4 / 15$ & \\
\hline 4. & $\mathrm{~T} 4 \mu \mathrm{g} / \mathrm{dl}$, Mean $(\mathrm{SD})^{*}$ & 9.96 (1.63) & $2.82 \quad(2.12)$ & 51.74 & $(22.28)$ \\
\hline \multirow[t]{2}{*}{5.} & TSH mU/L Mean & 3.1 & 79.7 & 0.1 & \\
\hline & Median & 3.5 & 83.2 & 0.09 & \\
\hline 6. & CD4/CD8 ratio Mean (SD) & $2.12(0.76)$ & $1.10 \quad(0.32)$ & 1.50 & $(0.55)$ \\
\hline 7. & Serum IL-5 (pg/ml) Mean (SD) & $7.55(1.50)$ & $14.66 \quad(3.34)$ & 24.77 & $(2.20)$ \\
\hline
\end{tabular}

Reference limits: T4 (4.5-12.5 $\mu \mathrm{g} / \mathrm{dl}) *$, TSH (0.25-5.0 mU/L)

* T4 to convert to SI units multiply by 12.87 HT: Hashimoto thyroiditis. 
When the ratio was correlated with other parameters (T4, TSH and AMc titre) within each group using the Spearman correlation test, no statistically significant correlation was found.

\section{Interleukin-5:}

The levels of serum IL-5 were $24.77 \pm 2.20 \mathrm{pg} / \mathrm{ml}$ $($ mean \pm SD) for GD, $14.66 \pm 3.34 \mathrm{pg} / \mathrm{ml}($ mean $\pm \mathrm{SD})$ for HT and $7.55 \pm 1.50 \mathrm{pg} / \mathrm{ml}($ mean $\pm \mathrm{SD})$ for the controls. Values of IL-5 were significantly higher for GD as well as HT $(\mathrm{p}<0.01, \mathrm{p}<0.01)$ compared to the controls when the KS test was applied. When the serum values of IL-5 were correlated with other parameters within each group of GD or HT using the Spearman correlation test, no statistically significant correlation was found in any group.

\section{DISCUSSION}

In the present study, we evaluated alterations in subsets of lymphocytes and interleukin-5 secreted by activated T-lymphocytes in the peripheral blood of patients with AITD. The study subjects included patients with hypothyroidism due to Hashimoto's thyroiditis and with hyperthyroidism due to Graves' disease. We found that the ratio of CD4/CD8 lymphocytes in patients with HT was significantly reduced compared to the control subjects but the difference was not significant between GD and controls. On the other hand, the values of IL-5 were significantly elevated in both HT and GD compared to those of controls. Neither the ratio nor IL-5 levels had any statistically significant correlation with T4, TSH or AMc in patients with either HT or GD.

Comparison of our findings with other studies on lymphocyte subsets is difficult due to the ethnic differences in their study populations from ours and different methodologies used. In addition, studies differ with regard to whether intrathyroidal lymphocytes or peripheral blood lymphocytes were used.

In a study on peripheral blood lymphocytes evaluating CD4 and CD8 subsets in GD and HT it was shown that the ratio was increased in GD and reduced in HT. ${ }^{14}$ In addition, they found correlation of levels with functional status but not with antibody titer levels. The ratio was reduced in HT in our study also but correlation with other parameters was not found.
Another study did not find any significant differences in CD4+ and CD8+ lymphocytes in the peripheral blood between HT patients and controls. ${ }^{15}$ Increased percentages of CD4 but normal CD8 lymphocytes were reported in GD as well as HT. ${ }^{16}$ In our study, the ratios were reduced in both disease entities but significant reduction was seen only in HT.

In another study evaluating intrathyroidal lymphocytes by Flow Cytometry (FC) in HT, CD4/ CD8 ratio less than 1 was associated with thyroid dysfunction. ${ }^{17}$ It was concluded that persistence of increased intrathyroidal CD8+ will predispose to hypothyroidism. Also, Type 1 response of lymphocytes with interferon- $\gamma$ (IFN- $\gamma)$ expression was found to predispose to occurrence of hypothyroidism in their study. Our study used peripheral blood lymphocytes in which the ratio was $<1$ in only $7 / 16$ cases, although all subjects with HT were frankly hypothyroid.

Our observations on IL-5 levels in the present study suggest a predominance of Th2 expression, the levels being much higher in GD compared to HT or control subjects. It has been earlier demonstrated that a shift to a Th2 driven cytokine pattern in thyroid tissues among GD patients results in high levels of $\mathrm{CD}^{5+} \mathrm{B}$ cells in hyperthyroid GD, and that these $\mathrm{CD}^{5+} \mathrm{B}$ cells are important in the production of autoantibodies. ${ }^{18,19}$ These antibodies stimulate the TSH receptor, causing thyroid cell hyperplasia and hyperfunction seen in GD. A possible role of these antibodies in protecting thyroid cells from apoptosis is also proposed..$^{20}$ Though titres of anti-microsomal antibodies have been shown to correlate with the degree of intrathyroidal lymphocytic infiltration in $\mathrm{HT},{ }^{21}$ this should have translated into correlation between levels of IL-5 and antibody levels, which was not found either in the present study or in other studies. ${ }^{10}$ This could be due to the use of the semiquantitative method for estimation of anti-microsomal antibodies and peripheral blood for evaluating lymphocytes in our study.

The elevation in IL-5 levels associated with reduction in lymphocyte subset ratios in the peripheral blood can only be explained by an intrathyroidal lymphocytic origin of IL-5. While predominance of intrathyroidal pathology in AITD is well known, ${ }^{22}$ this is further supported by the inconsistency between 
various studies on lymphocyte subset ratios in the peripheral blood.

Initiation of thyroid autoimmunity requires interplay of endogenous and environmental factors in a genetically susceptible individual, resulting in activation of thyroid-specific autoreactive T-cells. ${ }^{3}$ Among the environmental factors, excess iodine intake and salt iodization programs are considered to predispose communities to thyroid autoimmunity. This is based on evidence from experimental and epidemiologic studies, ${ }^{23,24}$ though the evidence is not conclusive. It is pertinent to mention that the study subjects resided in the national capital territory of Delhi, where a universal salt iodization program has been implemented for the past two decades. From this region, a study on goitre prevalence that was previously carried out on schoolchildren showed that, while Delhi has changed from iodine deficiency to sufficiency, nearly $28 \%$ of goitres were due to autoimmune thyroiditis (AIT). ${ }^{12}$ Hypofunction of the thyroid gland, both overt and subclinical, was seen in $28 \%$ of children with AIT, while only $3.6 \%$ had hyperthyroidism, and it was observed that higher urinary iodine levels correlated with thyroid dysfunction. It is possible that iodine plays a much larger role in the pathogenesis of HT than in GD through mechanisms such as increased immunogenicty of thyroglobulin, toxic destruction of thyroid cells by generation of free radicals or direct stimulation of lymphocytes. ${ }^{20}$ Consequently, increasing iodine intake among previously iodine deficient populations, as is the situation in India, may incite thyroid autoimmunity, particularly HT.

\section{CONCLUSIONS}

The present study showed reduction in ratios of CD4/CD8 lymphocytes in peripheral blood that could not be explained by the increased levels of IL-5 seen in AITD. Intrathyroidal lymphocytes and the cytokines secreted in the local environment play an important role in the pathogenesis of AITD. Iodine supplementation programs may have larger influence on pathogenesis of HT than on that of GD.

\section{REFERENCES}

1. Larsen PR, Davies TF 2002 Hypothyroidism and thyroiditis. In: Larsen PR, Kronenberg HM, Melmed S,
Polonsky KS (eds) Williams Textbook of Endocrinology, Saunders, Philadelphia; pp, 423-456.

2. Weetman AP, 1996 Recent progress in thyroid autoimmunity: An overview for the clinician. Thyroid Today 19: 1-9.

3. Gopalakrishnan S, Marwaha RK 2007, Juvenile Autoimmune Thyroiditis. J Ped Endocrinol Metab 20: 961-970.

4. Weetman AP, Mcgregor AM, 1994 Autoimmune thyroid disease: further developments in our understanding. Endocr Rev 15: 788-830.

5. Weiss A, 1990 Structure and function of T-cell antigen receptor. J Clin Inv 86: 1015-1022.

6. Davies TF, Larsen PR 2002 Thyrotoxicosis. In: Larsen PR, Kronenberg HM, Melmed S, Polonsky KS (eds) Williams Textbook of Endocrinology, Saunders, Philadelphia; pp, 374-422.

7. Mosmann TR, Sad S, 1996 The expanding universe of T-cell subsets: Th1, Th2 and more. Immunol Today 17: 138-146.

8. Weetman AP, 2000 Graves' disease. N Engl J Med 343: 1236-1248.

9. Roura-mir C, Catalfamo M, Sospedra M, Alcaide L, Pujol-Borrell R, Jaraquemada D, 1997 Single-cell analysis of intrathyroidal lymphocytes shows differential cytokine expression in Hashimoto's and Graves' disease. Eur J Immunol 27: 3290-3302.

10. Hidaka Y, Okumura M, Shimaoka Y, Takeoka K, Tada H, Amino N, 1998 Increased serum concentration of interleukin-5 in patients with Graves' disease and Hashimoto's thyroiditis. Thyroid 8: 235-239.

11. Hidaka Y, Okumura M, Fukata S, et al, 1999 Increased serum concentration of interleukin-12 in patients with silent thyroiditis and Graves' disease. Thyroid 9: 149-153.

12. Gopalakrishnan S, Singh SP, Walia RP, Jain SK, Ambardar VK, Sankar R, 2006 Prevalence of goiter and autoimmune thyroiditis in schoolchildren in Delhi, India, after two decades of salt iodisation. J Ped Endocrinol Metab 19: 889-893.

13. Landay A, Ohisson-Wilhelm B, Giorgi J, 1990 Application of flow cytometry to the study of HIV infection. AIDS 4: 479-497.

14. Covas MI, Esquerda A, Garcia-Rico A, Mahy N, 1992 Peripheral blood T lymphocyte subsets in autoimmune thyroid disease. J Investig Allergol Clin Immunol 2: 131-135.

15. Mazziotti G, Sorvillo F, Naclerio C, et al, 2003 Type- 1 response in peripheral CD4+ and CD8+ T cells from patients with Hashimoto's thyroiditis. Eur J Endocrino. 148: 383-388.

16. Ohashi H, Okugawa T, Itah M, 1991 Circulating T cell subsets in AITD: differences between treated and untreatead patients. Acta Endocrinol (Copenhagen) 125: 502-509.

17. Zeppa P, Marino G, Lepore M, et al, 2006 Flow cytometry phenotypization of thyroidal lymphoid infiltrate and 
functional status in Hashimoto's thyroiditis. Anal Quant Cytol Histol 28: 148-156.

18. Iwatani Y, Amino N, Kaneda T, et al, 1989 Marked increase of CD5+ B cells in hyperthyroid Graves' disease. Clin Exp Immunol 78: 196-200.

19. Heuer M, Aust G, Ode-hakim S, Scherbaum WA, 1996 Different cytokine mRNA profiles in Graves' disease, Hashimoto's thyroiditis, and non-autoimmune thyroid disorders determined by quantitative reverse transcriptase polymerase chain reaction (RT-PCR). Thyroid 6: 97106.

20. Fountoulakis S, Tsatsoulis A, 2004 On the pathogenesis of autoimmune thyroid disease: a unifying hypothesis.
Clin Endocrinol 60: 397-409.

21. Pandit AA, Vijay Warde M, Menon PS, 2003 Correlation of number of intrathyroid lymphocytes with antimicrosomal antibody titer in Hashimoto's thyroiditis. Diagn Cytopathol 28: 63-65.

22. Baker BA, Gharib H, Markowitz H, 1983 Correlation of thyroid antibodies and cytologic features in suspected autoimmune thyroid disease. Am J Med 74: 941-944.

23. Laurberg P 1994 Iodine intake - What are we aiming at? J Clin Endocrinol Metab 79: 17-19.

24. Bagchi N, Brown TR, Urdinavia E, Sundick RS, 1985 Induction of autoimmune thyroiditis in chickens by dietary iodine. Science 230: 325-327. 